\title{
Gravitational Catastrophe and Dark Matter
}

\author{
Mesut KAVAK
}

I have been working for a long time about basic laws of physics [1,2]. During this time I noticed, that gravity does not work as Newtonian [1]. Distance and gravitational force relation changes over distance. The attraction properties change for each point of free space, and have some limits. The attraction changes due to some values between $1 / r$ and $1 / r^{2}$ even for the existent furthest distance. This work aims to analyze and discuss this phenomenon.

\section{Introduction}

As we know, outer orbital objects rotate faster than inner orbital objects relatively in galaxies according to our observations. Namely an orbital object must take place in space according to $v=\sqrt{\frac{m G}{r}}$; but it was detected, that $v$ and $r$ are not related in this manner. Almost there is no exponential relation like this in galaxy size heavenly bodies.

\section{Attraction properties of gravity}

To say something, firstly I must tell something about nature of gravity as I discovered.

Matter emerges as a density over time by increasing acceleration as waves over free space being has time differences between any point of its free space. Space turns into particles and particles turn into space continual manner. Matter gains its mass by collecting space in a limited volume at light speed. During its emergence it constantly experiences potential difference since there are time differences in the other name emergence priority. There is a single work to create all the universe, and the work is done one by one for each point of free space and thus particles. By this way any point of the universe gets the same speed since the single work is done by the same speed as work-done is equal to kinetic energy. Total energy of matter is according to this creation work.

While matter experiences the potential differences, naturally there emerge some denser and low density and thus disordered or more ordered points. Disordered points want to be distributed on lower density or more ordered space points since have more stress. Matter gains its total energy because of the work done against this resistance of free space, that otherwise it would not be created since the existent smallest force can move the existent bigger mass magnitude at infinite speed.

The sliding space from disorder to more ordered point also takes the particles together with itself since the particles emerge over this sliding free space. Actual reason of gravity is this, and gravity is only distributed as waves over time along space.

\subsection{Attraction magnitude and distance relation}

If non-flexible collisions are handled, for two objects which move towards each other at light speed, (1) can be written after the collision.

$$
m_{1} c-m_{2} c=m c
$$

Here, if it becomes $m_{1}>m_{2}$, then the motion is going to be in + direction; otherwise it is going to be in - direction. For $m_{1}=m_{2}$, no motion can occur.

There are a few parts of some gravitational waves which are emitted by $m_{1}$ and $m_{2}$ masses placed at $B$ and $D$ points in Figure 1. The attraction emerges at $A_{1}$ and $A_{2}$ points for

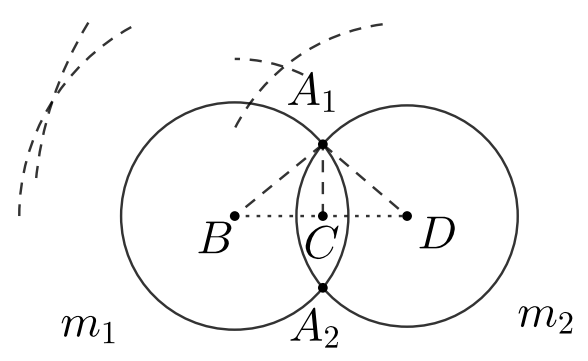

Fig. 1: Gravitational waves

this 2 dimensional wave section; therefore over the equality of $m_{a} c \cdot \cos (\alpha)-m_{b} c \cdot \cos (\beta)=m c$, it becomes (2),

$$
m=\left(\frac{m_{a} r_{1}}{k_{1}}-\frac{m_{b} r_{2}}{k_{2}}\right) j
$$

where $\cos (\alpha)=\frac{r_{1}}{k_{1}}, \cos (\beta)=\frac{r_{2}}{k_{2}}, k_{1}=A_{1} B, k_{2}=A_{1} D, r_{1}=$ $B C, r_{2}=C D, m_{a}$ and $m_{b}$ are reduced mass magnitudes at $k_{1}$ and $k_{2}$ distances, $j$ is a constant provides average particle collision amounts of waves since the smallest space parts can collide by different angles or do not collide that can change due to formation speed of light and can take 1 value, smaller or greater values than 1 but 0 .

Assume, that 0 dimensional densities of $m_{1}$ and $m_{2}$ over 3 dimensional densities are $m_{a}=\sqrt[3]{d_{1}} / k_{1}$ and $m_{b}=\sqrt[3]{d_{2}} / k_{2}$ where $\sqrt[3]{d_{n}}$ is 1 dimensional density over 3 dimensional $d_{n}$ density for $k_{1}$ and $k_{2}$ lengths. For total $m$ in 3D, over $2 \pi \mathrm{hm}$, (2) turns into (3),

$$
\Delta m=\left(r_{1} \sqrt[3]{d_{1}} \sqrt{k_{1}^{2}-\frac{r_{1}^{2}}{k_{1}^{4}}}-r_{2} \sqrt[3]{d_{2}} \sqrt{k_{2}^{2}-\frac{r_{2}^{2}}{k_{2}^{4}}}\right) 2 \pi j
$$

where $h=\sqrt{k_{1}^{2}-r_{1}^{2}}=\sqrt{k_{2}^{2}-r_{2}^{2}}$. This is for a single wave. I am not calculating gravity yet. Just analyzing attraction properties. Already at that point, there would emerge no attraction for the same magnitude two waves if you ignore time differences but a motion by gravitational torque; but this is not important. Here over Eq. (4),

$$
F t=\Delta m c
$$

you can calculate the attraction force and its way as (+), (-) or 0 being $t$ here is the time which the attraction emerges along 
a distance at light speed due to the constant wavelength of gravity I derived [1], and also this is not important now.

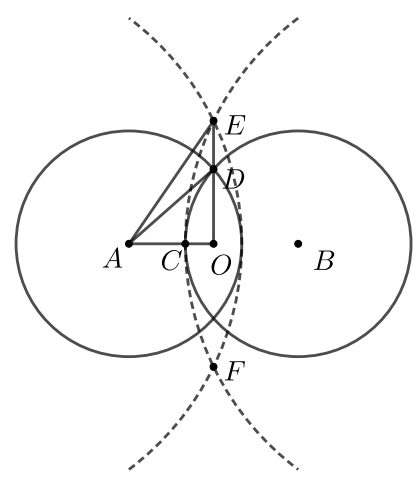

Fig. 2: Another representation of gravitational waves

Now let us handle the Fig. 2. There are two waves radiated from $A$ and $B$ points. The bigger wave parts are also radiated from a further distance than $A$ and $B$ by the same masses. Here $O C$ length is always fixed since it is the shortest distance which the attraction emerges along it for the gravitational wavelength when two waves are intersected.

For these definitions, if we remove the constants since they are not inclusive in change being mass is also fixed, and the other wave's values,

$$
F=\frac{O D}{A D^{2}}
$$

the attraction in $3 \mathrm{D}$ is basically dependent on Eq. (5) over Eq. (6) which is derived over Eq. (3) without the second wave since is not required,

$$
F=\sqrt[3]{\frac{3 m}{4 \pi A D^{3}}} \cdot \frac{1}{A D} \cdot 2 \pi O D
$$

where $\sqrt[3]{\frac{3 m}{4 \pi A D^{3}}}$ is 1 dimensional density of $m$ which the mass takes place at $A$ point, $\sqrt[3]{\frac{3 m}{4 \pi A D^{3}}} \cdot \frac{1}{A D}$ is 0 dimensional density of the same mass.

Since $O C$ is fixed, we can count it as 1 ; so it becomes Eq. (7) over $(A C+1)^{2}+O D^{2}=A D^{2}$.

$$
A C=\sqrt{A D^{2}-O D^{2}}-1
$$

Now if you assume that the same magnitude masses take place at a further distance, for this condition, the partial bigger waves on Fig. 2 emerge. $O C$ is still fixed. Even if it is fixed, as you can see, the other lengths exactly increase, and the rule is determined over Eq. (7). Over Eq. (7), we can easily state, that increase amount of $A D$ is always bigger than both $O D$ and $O A$; thus if distance between two the same masses increase gradually, Eq. (5) states, that the attraction decreases. If $A D$ converges to $O D$, then the attraction increases.

This condition is not a good news for scientists. It seems, Newtonian calculation can only work in an interval. Not for small distances as also is not for distant places. Already gravitational constant is completely experimental, and it can easily hide the actual functions which the gravity works over them. Namely it remains as faking up. We already must had feel re- sponsible about verifying it by another method. The motions in any universe are chaotic. They repeat in an interval; but you need more repeat and more time to draw the big picture; so gravitational attraction between stars or planets is misleading since is slow. Maybe you can detect some of the anomalies if they are too close to each other like deviation of Mercury. Namely it can be observed like the distance and attraction relation is over $1 / r^{2}$. For the better, for example electrons which have incredible spinning frequency are perfect references for motion as also the distance is short enough to detect anomaly relatively to Newtonian formula aside high speed.

\subsection{Time differences}

Everything is not okay. Even became more annoying; because the velocity of the outer orbital objects of galaxies must be much more slower according to these information. Namely if you assume that there is dark matter that there is no dark matter, the dark matter amount must be bigger than the estimated.

If the distance between two objects get bigger, the gravitational torque is going to increase more being more certain and sensible, detectable since gravity emerges at the two intersection point and the points have time differences. Emergence priority causes a potential difference between these points. Namely one of them emerges before and thus causes more attraction than the other one. Between two consecutive emerging smallest part of space, the time difference can be ignored but macro space.

While you are calculating orbital motion over classic method, you assume that whatever the emerging type of the centrifugal force, it must be equal to the attraction force; but it is not true completely. Actually you calculate it over static condition being two objects are standing mutually. Namely if you do not include the centrifugal force, the formula is gravitational attraction in linear way; but according to the above stated method, one of the intersection point emerges later.

If you think one point emerges earlier and state, that $F_{1}$ torque is applied at this point, being the second torque $F_{2}$ from the other point, it becomes $F_{1}>F_{2}$. Also it can be said, that energy of the medium namely wave must be conserved. When a space point gets lost since it has an emergence frequency and is not continuous at every point of itself, the energy must be transferred another point. It means, that while a wave is distributed on space over time, it cannot protect its shape. The increasing torque is stolen from the other second torque point. Even if again it is distributed on newly emerged space back, since to be of them before or after of the same two opposite $F t$ work as $+F t$ and $-F t$ causes displacement in a way (+) or (-) according to which one of them you used firstly even for the same values, it slides. Also rotation namely spin of the universe and thus in which way the masses take place affect the condition. By these ways, linear attraction force and thus momentum turns into angular momentum and attraction. There is no other method and alternative for this as required by conservation of energy. This is not a special phenomenon. The universe works in this way even if some times the result is going to be too small to detect.

As a result, the attraction which decreases over distance is supplied by the time difference-driven increased gravitational torque; but by which ratio?

\subsection{Calculation of the time differences}

The universe has an emergence frequency as also it has a spinning frequency as I derived both [1]. I calculated before the opposite force which free space applies to moving heavenly bodies in changing ratios according to the density of the objects, that for example Pioneer anomaly can be calculated 
over this [1] by natural holding capacity of free space; but to make a calculation for the time difference we must know how many space turned into particle.

We must add the extra slowing for the objects like Pioneer since gravitational torque is going to increase; so some of total momentum turns into angular momentum, and so it decelerates in linear way. Otherwise linear deceleration can be calculated over $-m a=V_{s} \cdot 1.35 \cdot 10^{-13} \cdot s$ as I derived [1] where $V_{s}$ is volume of the object, $m$ is mass of the object, $s$ is speed of the object and $-a$ is negative acceleration or deceleration.

Warning

Even if all particles get lost, the total mass, energy and frequency is conserved and thus can be used for such a calculation of the opposite force which is applied by free space; but like this calculation of time difference in galaxies, it is so hard. Actually for free space has no particle, the time difference between each cubic meter of the space becomes $1 / V$ being $V$ is the volume of the universe; but if a particle emerges, the density and thus the volume changes. And the amounts are so big. For example for 1 ton object, you need almost $10^{30}$ cubic meters free space according to my calculations; so think that $1 / V$ is disturbed by this value. You cannot say nothing changes by thinking observational distances that distance does not specify time. It does not work in that way. Space-time is flexible. We must determine some suitable ratios.

Actually it is good, that by the observation, we can calculate the total matter amount of the universe by calculating the deviation in speed and thus by calculating the time difference over this; but even so also we should find a different theoretical method. I do not know yet; but it seems almost at the amount of the distance between two object like Eq. (8),

$$
F_{G}\left(r_{1} t_{1}-r_{2} t_{2}\right)=m v^{2} / r
$$

where $F_{G}$ is Newtonian gravitational attraction, $r_{1} t_{1}-r_{2} t_{2}=$ $r_{0}$, and $F_{G} r_{0}$ is gravitational torque, $r_{0}=E F$ over Fig. 2 . Here $F r_{0}$ is not work-done. You can also write like $F t_{0}$ since is a multiplier. This equation is only representation to show why is like without exponential relation.

Gravity moves at light speed; thus also the gravitational latency must be included. Namely, actually outer orbital object store potential. If enough time has not passed yet, the acceleration changes. Otherwise it had gotten the maximum acceleration already. Namely for the life time graph of the galaxies in accordance with chaos, the upper and lower limits had already been determined. It cannot be so misleading.

\section{Warning}

\section{Conclusion}

These show us again, that the phenomenon is also chaotic, emerges in an interval in many different kind. These show us, that nothing can move away from each other after a point. There is a limit for this. They constantly wander around in a limited area. Just there is a flexible interval.

Space objects cannot move away from each other until infinity. This also explains why electrons do not fall into nucleus or fly off; because the phenomenon is not boosting. It is a transformation. Rotating effect of force increases; but this is for protecting the energy which the system holds.
Actually the relation between gravity and centrifugal force is $F_{G} \approx F_{C}$ since $F_{C}=-F_{G}$ requires zero energy and thus zero speed. One of them is bigger then the other one to emerge of matter as it was proven [3]. This requires Riemann surfaces and complex analysis since they cannot exist on the same axis together. Namely even if they are two main different variation of the same force, they must get different axis; but seems is not possible if you do not include complex plane. Complex plane allows it.

\section{References}

1. Kavak M. 2018, Complementary Inferences on Theoretical Physics and Mathematics. OSF Preprints. Available online: https://osf.io/tw52w/

2. Kavak M. 2016, On the Uncertainty Principle, American Journal of Physics and Applications, Vol. 4, No. 4, 2016, pp. 90-123. Available online: https://osf.io/t8zqw/

3. Kavak M. 2018, Short Note on Unification of Field Equations and Probability. Available online: https://osf.io/ptyvd/

Inference 